\title{
LA IMPORTANCIA DE UNA BUENA ESTRATEGIA DE FIJACIÓN DE PRECIOS COMO HERRAMIENTA DE PENETRACIÓN DE MERCADOS
}

\author{
THE IMPORTANCE OF A GOOD PRICING STRATEGY AS A TOOL OF MARKET \\ PENETRATION
}

\section{A IMPORTÂNCIA DE UMA BOA ESTRATÉGIA DE FIXAÇÃO DE PREÇOS COMO FERRAMENTA DE PENETRAÇÃO DE MERCADOS}

\author{
Por: CÓRDOBA SEGOVIA_Carlos Manuel, MORENO MONCAYO _ David Felipe
}

\begin{abstract}
Doctor en Globalización Económica y Sociedad de la Información, Universidad de Oviedo, España. Profesional en Finanzas y Relaciones Internacionales, Universidad Externado de Colombia. Profesor Tiempo Completo, Universidad de Nariño. Email: carcord13@yahoo.es, Colombia.

Profesional en Finanzas y Relaciones Internacionales, Universidad Externado de Colombia. Email: davidf994@hotmail.com, Colombia.
\end{abstract}

DOI: http://dx.doi.org/10.22267/rtend.171802.73

\section{RESUMEN}

Una empresa para estar a la vanguardia y al nivel de sus rivales, con bienes y servicios de alto valor agregado y notable especialización, requiere de una buena estrategia de fijación de precios (pricing) que sea atractiva para el cliente y, a la vez, rentable para la compañía. Este objetivo se puede alcanzar mediante técnicas de optimización financiera, centradas en el conocimiento al detalle de la estructura de costos de la empresa, que permitan aumentar la participación en el mercado a través de precios competitivos.

En este artículo se presentan diferentes estrategias de costos y de fijación de precios que, de acuerdo con la naturaleza de la empresa, se pueden utilizar para competir en el mercado de manera eficiente. Aunque, en la práctica, no existe una fórmula ideal de fijación de precios, la estrategia acertada será aquella que se adapte de la mejor manera al objetivo que quiera alcanzar la empresa en un momento determinado.

El propósito de este ensayo es exponer al lector las estrategias de pricing comúnmente utilizadas en el mercado, con el fin de demostrar su incidencia en los resultados financieros de las empresas y sugerir alternativas de toma de decisiones gerenciales. 
Palabras clave: fijación de precios, estructura de costos, competitividad.

JEL: G14, M31, L22.

\begin{abstract}
For a company to be at the forefront of its rivals, with high value-added goods and services as well as a significant expertise, must require a well-thought-out pricing strategy that is attractive to the client and profitable to the company. This objective can be achieved through financial optimization strategies focused on detail knowledge of cost structure that allow the increase in market share with competitive prices.
\end{abstract}

This article shows how different cost and pricing strategies that are related with the nature of each company can be used to compete efficiently in the global market. Although in practice there is no ideal formula for pricing, the right strategy will be the one that best suits the goal that the company wants to achieve at a given time.

The purpose of this essay is to expose to the reader the pricing strategies commonly used in the market, in order to demonstrate their impact on the financial results of companies and to suggest alternatives for management decision making.

Keywords: pricing, cost structure, competitiveness.

JEL: G14, M31, L22.

\title{
RESUMO
}

Uma empresa para estar à vanguardia e ao nível de seus rivais, com bens e serviços de alto valor agregado e notável especialização, requer de uma boa estratégia de fixação de preços (pricing) que seja atraente para o cliente e, ao mesmo tempo, rentável para a companhia. Este objectivo pode-se atingir mediante técnicas de optimização financeira, centradas no conhecimento ao detalhe da estrutura de custos da empresa, que permitam aumentar a participação no mercado com preços competitivos.

Neste artigo apresentam-se diferentes estratégias de custos e de fixação de preços que, de acordo com a natureza da empresa, pode utilizar para competir no mercado de maneira eficiente. Ainda que, na prática, não existe uma fórmula ideal de fixação de preços, a estratégia acertada será aquela que se adapte da melhor maneira ao objectivo que queira atingir a empresa num momento determinado.

O objetivo deste ensaio é expor as estratégias leitor de preços comumente utilizados no mercado, a fim de demonstrar o seu impacto sobre os resultados financeiros das empresas e sugerir as decisões de gestão alternativos.

Palavras chave: fixação de preços, estrutura de custos, competitividade.

JEL: G14, M31, L22. 


\section{INTRODUCCION}

En la actualidad, una estrategia de competencia empresarial fundamentada en la diferenciación de producto o servicio ha llevado a miles de empresas a ampliar su espectro de cobertura y a diversificar su portafolio, generando, cada vez, una mayor fricción entre los jugadores, quienes, a raíz de esta coyuntura, se han visto forzados a ofrecer un mayor valor agregado, lo que supone, generalmente, mayores costos de producción para satisfacer una demanda exigente y especializada.

Sin embargo, para estar a la vanguardia y al nivel de los grandes competidores, con bienes y servicios de alto valor agregado y alto grado de especialización, se requiere también de una estructura de costos que resulte atractiva para la empresa, por lo tanto, se debe buscar la forma de minimizar costos y, a la vez, aumentar los márgenes dentro del mercado de manera que, "para alcanzar una rentabilidad superior y sostenible, la fijación de precios debe formar parte integral de la estrategia y no debe ser una simple ocurrencia tardía" (Nagle y Holden, 1998).

Para esto, es imprescindible analizar múltiples factores al interior de las compañías, tales como: el talento interno, el conocimiento del mercado, la capacidad de innovación, la flexibilidad y la capacidad financiera, entre otros; variables mediante las cuales se llevarán a cabo las diferentes estrategias de precio - beneficio - costo como método de posicionamiento en el mercado.

Adicionalmente, para nadie es un secreto que el destino de las empresas depende de factores macroeconómicos, sectoriales, sociales, políticos, administrativos, aleatorios y demás. No obstante, la ventaja de una empresa sobre otra, dada las condiciones actuales de los mercados, en donde la competencia y la guerra de precios se dan por descontado, radica, en definitiva, en los pequeños detalles, en la capacidad de una compañía para ser más competitiva desde sus factores internos, puesto que el entorno que la rodea afecta por igual a cada uno de los competidores. Como afirman Rosa, Rondán y Díez de Castro (2013):

El aumento de la competencia en los mercados, la aceleración del progreso tecnológico, el incremento de la demanda de servicios, el aumento de la competencia extranjera, los cambios en el entorno legal y la incertidumbre económica han provocado que el precio sea utilizado cada vez con más frecuencia como arma táctica y estratégica.

Sin embargo, posiblemente, la diferencia no radique tácitamente en factores internos ni externos, también es necesario contar con algo de suerte que acompañe y armonice la actividad de la operación de cada compañía pero, sin duda alguna, dentro de los innumerables factores que pueden potenciar la actividad de cada empresa está la fijación estratégica de precios que puede hacer la diferencia a la hora de determinar el rumbo de una compañía exitosa, frente a otra que por azar o mala gestión la lleven al fracaso. 


\section{SOBRE LAS ESTRATEGIAS DE COSTOS}

Una de las diferencias principales del costeo de servicios frente al costeo de otras actividades productivas, es que los primeros en su mayoría no presentan un costo material dado a su naturaleza intangible, en donde el costo interno yace en gran medida del valor agregado del servicio, que a su vez es producido por la capacidad de análisis y el conocimiento de quien lo presta recibiendo una remuneración como contraprestación.

Sin embargo, independientemente de si la naturaleza de los costos sea material o no, la estructura de los costos permanecerá inalterada en la mayoría de las actividades del sector económico. Cualquiera que sea la estructura de costos de una empresa indiferentemente de su sector, estará conformada en parte por unos costos fijos y lo restante por costos variables, que a su vez serán únicos para cada empresa según sus necesidades y sus preferencias.

La distribución de la participación de los tipos de costos dentro de la estructura de una compañía, estará condicionada por factores tales como el tipo de sector en el que se desempeñe, la voluntad gerencial o administrativa, la coyuntura económica, entre otros. De ahí, la importancia a la hora de la conformación de la estructura de costos, de balancear lo más acertadamente la ecuación, con el fin de lograr una estructura funcional y más importante aún, rentable y sostenible en el horizonte de tiempo.

$>$ Tipos de costos:

- Costos fijos: los costos fijos son la columna vertebral de cada compañía, de estos se desprende el funcionamiento de la actividad principal de la empresa. Este tipo de costos se definen como (Pindyck y Rubinfeld, 2009) "el coste que no varía con el nivel de producción y que solo puede eliminarse cerrando" la empresa. Los costos fijos no son sensibles a cambios en la actividad productiva de la empresa, permaneciendo invariables a lo largo del tiempo y adicionalmente suelen ser periódicos mensuales, semanales, anuales, etc.

- Costos variables: Contrario a los costos fijos, los costos variables tienen un comportamiento sincronizado con la actividad productiva, es decir, a medida que la actividad productiva incrementa por lo general estos también lo hacen y viceversa.

Generalmente, los costos tienden a comportarse de manera lineal, sin embargo, en la teoría microeconómica los costos tienen una tendencia inicial de rendimientos crecientes y una final de rendimientos decrecientes sujetos a una serie de supuestos. Por otra parte, en un horizonte temporal muy breve todos los costos tienen un carácter fijo en cambio, cuando se consideran periodos largos, muchos costos fijos se pueden considerar como variables (Pindyck y Rubinfeld, 2009).

Una estrategia de costos exitosa dependerá entonces del mejor uso posible de los recursos disponibles, donde es preciso gestionar los costos de operación de manera 
óptima, buscando minimizar los costos totales para maximizar la utilidad de la compañía. En teoría, toda empresa realiza periódicamente un seguimiento sobre su operación como parte normal de la rutina de la gestión empresarial, dentro de la cual se evalúan las hojas de ruta a seguir, los planes estratégicos, los rendimientos esperados y demás. Al interior de cada evaluación de gestión y resultados, se valoran los esquemas de costos e ingresos vigentes, como parte de la planificación estratégica de las compañías. Todo lo anterior, con el fin de determinar las directrices encaminadas a la toma de decisiones frente a las estructuras de costos, teniendo en cuenta todos los factores tanto externos como internos que rodean la coyuntura y la actividad empresarial.

Una gestión óptima de los costos deberá tener en cuenta los siguientes aspectos:

$>$ Productividad

$>$ Curvas de aprendizaje y nivel de capacitación del personal

$>$ Choques y cambios tecnológicos

$>$ Costos operativos prescindibles

$>$ Costos operativos imprescindibles o estratégicos

$>$ Naturaleza y origen de los costos

La productividad es un factor esencial dentro de una estrategia de costos dado que existe un alto grado de correlación entre su variación y la de los costos variables. Un incremento en la productividad repercutirá positivamente en una reducción de los costos variables.

Una valoración correcta de las curvas de aprendizaje y el nivel de capacitación de los miembros de la compañía, es necesaria para cuantificar la capacidad productiva de la "mano de obra" de la empresa. Un mayor nivel de cualificación incidirá positivamente sobre la productividad, los costos variables y consecuentemente sobre los costos totales.

La capacidad de adaptación frente a los cambios tecnológicos puede significar la diferencia a la hora de ser competitivos en cualquier mercado. La adaptación es casi tan importante como la renovación tecnológica en cualquier sector de la economía, ya que un mayor nivel de tecnología implica mayor productividad por lo tanto, menores costos totales y mayor competitividad.

Por otro lado, la evaluación precisa y progresiva de los costos operativos y no operativos le permite a la empresa tener mayor flexibilidad a la hora de adaptarse a la coyuntura de los mercados; el conocimiento de los costos fijos sobre los cuales se puede prescindir es vital para una correcta dinámica de cualquier empresa. Los recortes o eliminación de costos permiten sortear los ciclos económicos con mayor facilidad ya que propician un margen de maniobra y otorgan flexibilidad financiera. No basta con tener un respaldo contable sobre los costos sino conocer a profundidad la capacidad de gerencia que se tiene sobre los mismos. En este caso se habla de los costos como un recurso sacrificado para alcanzar un objetivo específico que se amolde a una situación determinada.

Finalmente, la naturaleza o el origen de los costos son de suma importancia a la hora de determinar la estructura de costos de cualquier compañía. Cuando la denominación de 
los ingresos difiere del origen de los costos, hay que generar estrategias que inmunicen la actividad empresarial de posibles choques y externalidades que puedan afectar una normal operación comercial. Herramientas como las coberturas con derivados para el tema de divisas o futuros y opciones sobre materias primas, pueden ayudar a mejorar y proteger la gestión empresarial.

En algunas ocasiones puede darse la situación de que la estructura de los costos esté basada en la calidad de los ingresos, generando que ante una eventualidad que altere el normal cauce de la actividad empresarial, la estabilidad de la empresa quede expuesta al riesgo de la coyuntura, por lo que si bien es correcto fijar la estructura de costos con base en la calidad de los ingresos es necesario establecer políticas desde los costos que permitan hacer frente a tales cambios.

$>$ Caso empresarial (acercamiento teórico)

- Avianca Holdings:

\section{Contexto Avianca Holdings}

Una de las más grandes compañías del sector aeronáutico en todo el continente americano con presencia internacional en 8 países del continente y con más de 90 años de experiencia en el mercado. Actualmente, Avianca es la principal aerolínea del país tanto en número de operaciones como en ingresos netos. Entre enero y diciembre de 2015 los ingresos operacionales de la empresa sumaron US\$4,36 billones y la utilidad neta ajustada fue de US\$67,8 millones con una contracción porcentual del 14,1\% con respecto al periodo inmediatamente anterior. (Revista Dinero, 2016).

\section{Avianca y el problema de costos}

La coyuntura macroeconómica mundial y regional puso a prueba la capacidad gerencial y de respuesta tanto de los directivos como la de los encargados de la planificación financiera de la empresa. Los cambios tanto en la estructura de ingresos como de costos, generaron fuertes ajustes al interior de la empresa como respuesta a los choques externos e internos a los que se enfrenta el holding empresarial en diferentes países.

El punto neurálgico del impase financiero de Avianca radicó principalmente en la incompatibilidad de la estructura de costos frente a la estructura de los ingresos, dadas las condiciones del momento.

Por un lado, parte de los costos fijos de la empresa están denominados en dólares (combustible, repuestos de aviones, etc.), por lo que al estar atados a una moneda fuerte la probabilidad de que permanezcan estables a futuro, en términos cambiarios, es alta mientras que, a su vez, están expuestos a la volatilidad del mercado de commodities. Por otro, los ingresos del conglomerado empresarial están denominados en la moneda local de los diferentes países donde la compañía tiene sus hub de operación como consecuencia; 
los ingresos están atados a las diferentes tasas de cambio de donde la empresa tiene operaciones, quedando expuesta al riesgo de tasa de cambio.

Son tres los factores que han incidido sobre las finanzas del holding, el primero de ellos tiene que ver con la caída de los precios del petróleo, impactando positivamente sobre la estructura de costos fijos dependientes de los derivados de esta materia prima. El segundo tiene que ver con la depreciación de las monedas de los países donde la compañía tiene operación, lo cual contrarrestó el efecto de la reducción de los precios del petróleo, ya que la importación de las materias primas se encareció radicalmente. Por último, los indicadores macroeconómicos de los países de la región se vieron fuertemente deteriorados provocando una alta contracción de la demanda, que sumado a los demás factores, provocaron un desequilibrio en la situación financiera de la compañía socavando las finanzas internas.

Ante esto, durante el primer trimestre del 2016, la compañía redujo en 10,9\% los costos operacionales beneficiados por los bajos precios del petróleo sin embargo, los ingresos totales presentaron una reducción del 9,9\% como consecuencia del impacto de la devaluación y de la reducción de la demanda.

Lo anterior, es un ejemplo de como una buena estructura de costos permite sortear con mayor facilidad fenómenos económicos a nivel interno teniendo en cuenta que las condiciones macroeconómicas o coyunturales tienen el mismo impacto para cada uno de los jugadores del mercado. De ahí, la importancia de hacer la diferencia desde la gestión gerencial y el manejo de costos. No obstante, una buena estrategia de fijación de precios tendrá un mayor impacto sobre la rentabilidad de la empresa, como se verá más adelante.

\section{ESTRATEGIAS DE FIJACIÓN DE PRECIOS}

La consolidación de una buena estrategia de pricing es de suma importancia para cualquier actividad empresarial como método efectivo de planificación financiera, Robert J. Dolan (1995) ilustra la repercusión de una correcta fijación de precios de la siguiente manera:

Acercarse al precio "correcto" puede tener un tremendo impacto. Incluso pequeñas mejoras pueden producir resultados significativos. Por ejemplo, para una empresa con márgenes de beneficio del 8\%, una mejora del 1\% en el precio de realización, suponiendo una constante de las ventas en unidades de volumen, impulsaría el margen de la compañía en un 12,5 \%. Por el contrario, una reducción de los costos fijos en un 1\% sólo conduciría a un incremento en los beneficios de 4\%, por esa razón cualquier paso que se dé hacia un mejor pricing puede contribuir enormemente.

Un ejemplo de esto es el caso de Continental Airlines, que en 2001 manejaba un flujo alrededor de 44 millones de pasajeros en todo el mundo. El costo promedio de sus tiquetes de avión rondaba los 193 USD (a precios constantes), sobre el cual hizo 
un incremento equivalente a 2 USD o 1,04\% más por tiquete. Este pequeño incremento transformó las pérdidas en ganancias con una modificación en el precio casi imperceptible (Massachusetts Institute of Technology, 2010).

$>\quad$ Esquemas clásicos de fijación de precios

- $\quad$ Pricing basado en costos (Cost - Based pricing):

Este esquema de fijación se basa en la medición de los costos internos de las firmas tanto fijos como variables. Inicialmente, se espera que el precio del producto o servicio cubra los costos de producción y, además, genere un rendimiento a partir de un margen adicional que produzca dicha utilidad. El pricing basado en costos se representa por la siguiente ecuación:

\section{(1) Precio $=(1+$ Margen de ganancia $)($ Costo variable unitario + Promedio costos fijos)}

Para la implementación de un "cost based pricing" es necesario evaluar factores tales como la precisa medición interna de los costos, la coyuntura del mercado y el tipo de industria.

Un conocimiento impreciso de los niveles de costos exponen a las compañías a incurrir en pérdidas, producto de la deficiente medición de sus cuentas internas. Desconocer costos adicionales, atípicos o temporales puede llevar a implementar una mala estrategia de precios en detrimento de los intereses empresariales.

En una economía de escala los costos están en función de las ventas, las cuales a su vez están en función de los precios, generando una relación circular. Suponer una compañía cuyos costos se reduzcan en función de sus ventas. En un eventual caso de que la empresa reduzca sus volúmenes de ventas, como consecuencia de una contracción de la demanda agregada, ¿es posible para la compañía aumentar los precios para contrarrestar el efecto del aumento de costos?, Posiblemente no. Ahora, suponer el caso inverso, si la empresa aumenta sus volúmenes en teoría deberían reducirse progresivamente sus costos, pero si se trata de una economía de pequeña escala el efecto sobre la reducción de costos será mínima al igual que su impacto (Massachusetts Institute of Technology, 2010a).

El pricing basado en costos resulta ineficaz en industrias donde existen altos costos fijos y los costos marginales son cercanos a cero debido a la dificultad de distribuir la carga de los costos.

Por último, la fijación de precios con base en costos puede conducir a ignorar el valor intrínseco del producto o servicio generando que el valor en el mercado sea menor a su verdadero valor agregado.

- $\quad$ Precio basado en el consumidor (Costumer based pricing): 
En la práctica existen varios tipos de costeo basado en el consumidor, sin embargo, la clasificación general se hace con base en el tipo de consumidor final que se beneficiará del producto o servicio. Si el consumidor final es una persona natural se habla de un sistema B2C (Business to Consumer). Por el contrario, la relación de compraventa se desarrolla entre personas jurídicas se habla de un sistema B2B (Business to Businees).

En el sistema B2B las fuerzas de venta permiten a los agentes de compra determinar los precios finales de los productos o servicios. Generalmente, esta situación viene condicionada por el miedo de los proveedores a perder clientes estratégicos, lo que conlleva a incurrir en pérdidas dado que se tiende a vender por debajo del costo de producción. En el modelo B2B las dos partes involucradas en la transacción son empresas. En el sistema B2C, el productor/prestador del producto o servicio regala un producto de alto valor agregado o un complemento por temor a una reacción adversa del cliente.

El principal problema que generan estos sistemas de pricing es que las empresas dejan sus estrategias de precios a merced de los caprichos de los clientes, perdiendo independencia sobre parte de la planificación financiera. "En lugar de preguntarse lo que pagarían sus clientes, las empresas deben preguntarse cuáles son los precios a los que pueden convencer a sus clientes soportados por el valor de su producto" (Massachusetts Institute of Technology, 2010a)

- $\quad$ Precio basado en la competencia (Competition based pricing):

Las compañías que hacen uso de este tipo de fijación de precios, proyectan su estrategia con base en las decisiones tomadas por la competencia dentro de un sector y segmento determinado. No existe una política específica de precios relacionada con el valor de sus productos o servicios, su estrategia únicamente refleja las decisiones en materia de pricing tomadas por sus competidores. Generalmente, las empresas que hacen uso de este tipo de estrategia, están en busca de una cuota de mercado generada a través de un menor precio frente a sus competidores y es mantenida hasta la consecución del objetivo.

Este tipo de estrategia conlleva inherentemente una serie de inconvenientes para las compañías, tales como ignorar su propia propuesta de valor como factor diferenciador, generan guerra de precios dada la similitud de las políticas de mercado, y no crean certeza sobre la rentabilidad, ya que una cuota de mercado mayor no necesariamente garantiza mayores márgenes de ganancias.

$>$ Otros esquemas de fijación de precios:

- Valor económico para el consumidor (Economic Value to Consumer):

Este tipo de estrategia se basa en el principio donde un comprador adquirirá el producto/ servicio, únicamente, si para él su valor intrínseco es mayor que el de la alternativa más cercana, o cuando la Utilidad de $\alpha$ lfa es > Utilidad de ßeta. La utilidad del producto/ 
servicio dependerá entonces del valor que represente para el consumidor menos su precio, otorgando la siguiente relación (Massachusetts Institute of Technology, 2010b):

(2) Valor $\alpha$ - Precio $\alpha \geq$ Valor $\beta$ - Precio $\beta$

Reordenando se obtiene:

(3) Precio $\alpha \leq$ (Valor $\alpha-$ Valor $\beta$ ) + Precio $\beta$ Precio $\alpha \leq$ Valor de diferenciación $\alpha \beta+$ Precio $\beta$

La diferenciación entre el producto $\alpha$ y $\beta$ permite resumir el precio de un producto/ servicio como:

(4) Precio $\alpha \leq$ Valor de diferenciación $\alpha \beta+$ Precio $\beta$

Por lo tanto, una firma deberá fijar un precio similar o por debajo del de sus competidores, más el valor de diferenciación que su producto/servicio tiene para los consumidores, de manera que quien adquiera el producto lo prefiera por encima de los demás disponibles en el mercado, a pesar de tener precios finales diferentes.

Una práctica de implementación de un pricing basado en el valor agregado, debe identificar cuáles son sus competidores más cercanos y calcular sus precios, debe además identificar las fuentes potenciales de valor de diferenciación, y debe calcular qué tanto valor agregado está creando para poder definir una estrategia efectiva.

Este esquema de fijación se recomienda i) cuando los precios de la competencia son de conocimiento popular y tienen un valor concreto, ii) cuando la diferenciación del valor agregado del producto es fácilmente perceptible y, iii) cuando es posible transmitir al consumidor el factor diferenciador. De lo contrario, puede ser llevado al mercado un producto que sea un fracaso como consecuencia de su elevado precio y de la indiferencia del consumidor hacia el supuesto factor diferenciador.

\section{CONCLUSIONES}

En la práctica no existe una fórmula ideal de fijación de precios, la estrategia acertada será aquella que se adapte de la mejor manera a la naturaleza de lo que sea que se quiera transar en un momento determinado. La diferencia entre un buen sistema de pricing y uno malo, yace de cómo se realice el análisis de mercado, económico, coyuntural, sectorial e interno que rodea a la compañía. Por tanto, la idoneidad de un sistema no está predeterminada para ningún tipo de compañía ni sector, sino que, por el contrario, subyace de la experticia del planificador y del equipo financiero de cada empresa, así como del objetivo que se quiere obtener; bien sea una cuota determinada de mercado, fidelización de los clientes, etc.

Innumerables casos empresariales como el de Avianca o el de Continental Airlines, dan fe de la importancia en un buen sistema de pricing ya que puede ser determinante en el 
rumbo de cualquier firma a la hora de alcanzar buenos resultados financieros o poner en aprietos al equipo financiero, como consecuencia de las pérdidas generadas.

En síntesis, un buen sistema de fijación de precios no garantiza el éxito empresarial, ya que los resultados no sólo dependen de este tipo de estrategias de planificación, también es necesario contar con condiciones de favorabilidad del mercado, una innovación constante y una buena planificación financiera en general, que permita un buen desarrollo de la actividad empresarial. Sin embargo, los académicos y casos empresariales demuestran que un buen esquema de fijación de precios puede generar ventajas significativas que pueden ser determinantes en el rumbo de cualquier compañía.

\section{REFERENCIAS}

(1) DOLAN, Robert J. (1995). How do you know when the price is right?, Harvard Business Review. Pag1. (en linea). Disponible en: https://hbr.org/1995/09/how-do-you-know-when-the-price-is-right.

(2) MASSACHUSETTS INSTITUTE OF TECHNOLOGY (2010a). Pricing beyond de 3 CS, Pag 1 - 4 . (en línea). Disponible en: https://ocw.mit.edu/courses/sloan-school-of-management/15-818-pricing-spring-2010/ lecture- IT15_818S10_lec01.pdf

(3) MASSACHUSETTS INSTITUTE OF TECHNOLOGY (2010b). Pricing economic value to customer. Pag 1 - 6 (en linea). Disponible en: https://ocw.mit.edu/courses/sloan-school-of-management /15-818-pricingspring-2010/lecture-notes.

(4) NEAGLE, Thomas T.; HOLDEN, Reed K. (1998). Estrategia y tácticas para la fijación de precios. Pag22. España: Ediciones Juan Granica.

(5) PINDYCK, Robert S.; RUBINFELD, Daniel L. (2009). Microeconomía. Madrid: Pearson Eduación, S.A.

(6) REVISTA DINERO (2016). Los ingresos totales de Avianca disminuyeron 14\% en cuarto trimestre de 2015 (en línea). Disponible en: http://www.dinero.com/empresas/articulo/resultados-de-avianca-cuartotrimestre-de-2015/220894

(7) ROSA, Isabel María; RONDÁN, Francisco Javier; DÍEZ DE CASTRO, Enrique Carlos. (2013). Gestión de Precios. Pag29. Madrid: ESIC Business Marketing School. 